\title{
ANALYSIS OF THE COOPERATION MODEL BETWEEN INDUSTRY, UNIVERSITY, RESEARCH CENTER AND GOVERNMENT FOR THE SCHEDULING OF TECHNOLOGIES
}

\author{
Jayson Luis da Silva Ribeiro \\ Federal University of ABC \\ Santo André, São Paulo, Brazil
}

\author{
Anderson Orzari Ribeiro \\ Federal University of ABC \\ Santo André, São Paulo, Brazil
}

\author{
Ricardo Gaspar \\ Federal University of $\mathrm{ABC}$ \\ Santo André, São Paulo, Brazil
}

\begin{abstract}
Member of a globalized economy focused on innovation, the ABC region of São Paulo state represents an important pole of national economic development. With a strong industrial presence and a relevant role in the production of goods and services, the region has important research, development and innovation (R\&DI) hubs, including universities and governmental / industrial support agencies. Although there are incentives for the development of new technologies in basic research, insufficient effort has been done to foster innovative research and development projects in the stage of scheduling (TRL4 +). Based on the concepts of the triple helix, this research had the objective of evaluating the cooperation models existing in 13 companies that worked in the development of new technologies and presented interruptions in the development of their R\&DI projects. The selection of the participating companies was made by the adequacy to the theme, availability and adoption of development and innovation practices. The method was applied through the application of a semi-structured questionnaire, the use of interviews to deepen the data collected in the first phase, at the same time, interviews were made with regional development agencies, including economic development secretariats of the municipal government. An analysis of existing and in development innovation incentive programs and their possible adaptation to the studied research problem was carried out. The results of the present research point to the existence of the so-called death valley of scientific research (TRL4 +) in the region, pointing out as one of the main causes for this fact, the inherent costs of acquiring and maintaining a scientific research infrastructure in micro and small enterprises (MSE) analyzed. The research points to the need to review the existing models of cooperation and development of new technologies, positioning the sharing of R\&DI infrastructures in phase TRL4+ as a possible solution to strengthen the regional and national economy and increase the efficiency of research conversion rates technologies for the market.
\end{abstract}

Keywords: infrastructure r\&d; valley of death; technology readiness level (trl); triple hélix; innovation

\section{INTRODUCTION}

The concept of Valley of Death (stages of the method of evaluating the maturity level of a technology - Technology readiness level - TRL) is object of study in the field of knowledge and management of innovation. These steps refer to the need to raise resources for the development of prototypes for technology scaling, which represent a critical point for the development of products and services [1].

Brazil has been making efforts to foster innovation through the strengthening of research practices through the National Innovation System (SNI). Although there are some efforts made for the promotion of R\&DI (Law of Innovation, Law of Good, Law of the National Fund for Scientific and Technological Development - FNDCT), further studies are necessary to understand the variables involved in abandoning research during the stages of new technology. And the difficulty to overcome this stage of the development of new products and services is even more relevant when it comes to micro and small enterprises (MSE).

According to the general law of micro and small enterprises (2006), MPE are legal entities that have annual revenues of less than or equal to $\mathrm{R} \$ 3,600,000.00$. Filion (1990) [2], defines micro and small companies as legal entities that depend mainly on a source of internal capital for their growth. Similarly, according to data from SEBRAE (2017) [3], based on its analysis report on the register of employees and the unemployed (CAGED), small and medium-sized Brazilian companies represent one of the most important national economic pillars through job creation and local economy.

Although it is a sector of great relevance in the national economy, the MSE market is prone to specific bottlenecks especially regarding the costs related to the acquisition of infrastructure for research and development of a new technology. Pavitt et al. (1987) [4] defines sectorial patterns of innovation, categorizing companies into groups according to the technological opportunities identified through Research, Development and Innovation (R\&DI) projects and their 
particularities and limitations. In this context, SMEs are more dependent on sources of external capital for the development of their projects.

Schumpeter (1934) [5] defined innovation as a fundamental element for economic development with the capacity to substantially change relations between producers and consumers. The technological innovation, according to Neto and Longo (2001) [6], refers to how the market has absorbed this technology. Brown (1993) [7] defines innovation as "a new product, process or system that has the potential to create an entirely new market or change an existing market".

Through this innovation process, companies can constantly develop technologies and create processes to share and improve knowledge, techniques and production methods within the production chain. In addition to the transfer of knowledge, companies seek partnerships for the transfer of technologies in order to ensure that the development of this new product becomes possible, a step that Barbiere (1990) [8] calls technology transfer. According to Neto and Longo (2001) [6], "Trade, technology transfer and technological innovation are, by their own peculiarities, different stages of the same process, more comprehensive and complex, beginning with the basic / applied research phase and concluded when innovation is carried out (p.96)"

Technology usually goes through a non-linear innovation process flow that involves several stages, starting with basic or applied research, passing to a stage of technological development, and finally passing through a prototyping and system development stage. Thus, universities and institutions of Science and Technology - ICT have an extremely important role in generating knowledge for the development of a technology, especially in the initial stages of this flow.

The current problem with innovation and technology development is that some of the idealized researches are not capable of reaching its final stage, that is, its commercialization. Although part of this rate of abandonment of new research is related to technical unfeasibility, research abandoned due to lack of physical and financial resources represents a significant loss of knowledge and technologies improvement in the market.

Faced with this question, the present work aimed to identify the causes of abandonment of research projects during the stages of scheduling in the new product or service development. It investigated abandoned projects due to lack of resources and adequate infrastructure, as well as evaluated the role and relevance of the triple helix (University / ICTs, government and companies) as a tool to reduce this type of events.

The concept of Death Valley and its relation with the development of new technologies for an assessment of costs is important for new technologies. We looked at a possibility of infrastructure sharing as a way to reduce this kind of gap.

With a focus on the research and development field, especially regarding innovation in micro and small companies, the set of R\&DI infrastructure opportunities for the scaling of new technologies in the context of the National Innovation System (SNI).

\section{METHOD}

We interviewed 13 chemical companies that worked on the development of new products in the region of the great $\mathrm{ABC}$ Paulista. The selection process of the individuals composing the sample was based on the following factors:

- Acting in the region of the great $\mathrm{ABC}$ in the state of São Paulo;

- Participate in the chemical segment;

- Investing efforts and resources in the development of new technologies;

- Previous participation in a consulting project to promote regional innovation.

For purposes of comparison and analysis of the results, the participating companies were divided into two groups: a) Group of companies with a tax frame-work such as micro or small enterprise (MSE), with a turnover of less than or equal to R \$3,600,000.00 and up to 99 employees, and; b) Group of companies with a fiscal environment such as medium or large companies, including multinationals and corporations (S / A), with a turnover greater than or equal to $\mathrm{R} \$ 3,600,000.01$ and above 100 employees.

The initial data collection was performed through an electronic questionnaire with seventeen questions applied to Brazilian entrepreneurs, investigating the relevance of a R\&DI infrastructure in the development of new technologies. After that, in a second stage of data collection, three relevant cases were chosen regarding the current model of cooperation between universities, research centers and government for the scheduling of new technologies.

The data resulting from the present research were statistically analyzed, composing a current scenario about the mortality in the development of new products in the companies analyzed and its relation with the possible absence of adequate research infrastructure for the development of technologies and innovation.

With the purpose of organization and rational analysis of the data, the questionnaire was divided into four axes of information, as described below:

- Axis 1 - Basic characteristics and delimitation of the public;

- Axis 2 - Offer of new products and innovation:

- Axis 3 - Knowledge and use of partnerships; 
- Axis 4 - Infrastructure for technology development.

The first axis refers to the obtaining of basic characteristics, aiming the delimitation of the target public. Issues related to the area of activity of the company, fiscal framework and other profile delimiters were discussed.

The second axis refers to the offer of new products and innovation. Analyzing if investments were made in the development of technologies and new products for the market, including patent registration and innovative ambience.

The third axis is called knowledge and use of partnerships and analyzed the company's participation in government programs to foster innovation and obtain lines of credit for research.

The fourth axis analyzed aspects related to infrastructure for the development of technologies, addressing issues related to the purchase and costs of R\&DI equipment and their impact of technology development.

This article will focus on research axes 3 and 4 of the scientific study carried out. The other axes will be approached in a timely manner.

\section{MAIN RESULTS AND CONCLUSION}

Analyzing the data, it was possible to notice that $23 \%$ of the companies had problems in the finalization of the R\&DI projects initiated, of which the totality is composed of MSE.

After analyzing the existence of interruption in the development of new technologies by $23 \%$ of the companies surveyed, it was sought to address this reality more deeply identifying the causes for this abandonment of research. Among the answers obtained, the following variables were verified: a) lack of financial resources; b) lack of knowledge of the processes; c) lack of skilled labor; d) lack of research and development infrastructure; e) lack of theoretical knowledge; f) short deadlines; g) market change; h) location of part of the R\&DI infrastructure outside the national territory (United States and Germany); i) need to maintain productivity.

The most mentioned items as an integral part of the difficulties in the development of new technologies were the lack of skilled labor with $36 \%$ of the answers, followed by a lack of R\&DI infrastructure with $24 \%$ and lack of financial resources with $16 \%$ respectively.

An interesting fact to note is that although it is a determining factor in the development of a technology, the lack of financial resources is not the main difficulty addressed by the companies analyzed in this study. This demonstrates that more than cash on hand, the companies surveyed need skilled professionals and appropriate equipment for the development and scheduling of a new technology.

The identified scenario corroborates the hypothesis formulated that there are some gaps in the actual model of cooperation between the triple helix agents, as well as, the inherent costs of obtaining and maintaining a R\&DI infrastructure is a relevant factor for the abandonment of researches of new technologies in the chemical sector of MSE analyzed in this study.

The results reinforce the idea that by sharing a R\&DI infrastructure, MSE could benefit from greater efficiency in converting basic research into new technologies for the market, since this alternative directly addresses the three main bottlenecks raised by micro and small entrepreneurs.

With regard to the lack of skilled labor, through the sharing of infrastructure by institutions of science and technology and the government, MSE would have the support of technical specialists in each equipment. The project management itself is still in charge of the company, but the operationalization of the activities will count with the support and knowledge of a laboratory technician or researcher of a partner institution. Issues regarding patents, research secrecy and intellectual property should be well delimited and established for the success of this initiative.

Continuing the analysis of the factors relevant to the interruption of research projects, the second variable most mentioned by the entrepreneurs was the lack of research infrastructure itself, which demonstrates a lack in the appropriate infrastructure segment for the development of new technologies. Although there are some lines of government funding to obtain equipment, the risk associated with the development of innovative products may inhibit the completion of projects with disruptive innovations. By sharing research and development infrastructures, the science and technology institutions are part of the risk inherent in this process, being responsible for the cost of infrastructure and specialized technical capital for management and maintenance.

Considering the point of view of Science and Technology Institutions, in addition to the high costs of purchasing some equipment, R\&DI laboratories face great challenges in terms of balance and financial sustainability. Expenses with the management of the laboratory environment, including costs with specialized technical labor consumables and equipment maintenance, represent a considerable share of its operating costs. By dissolving these costs in various development projects, these institutions can enjoy greater efficiency in managing the operations and their core business.

As for the third aspect relevant to the failure of the researches in the analyzed companies, it was verified that the lack of financial resources can directly impact on the abandonment of these researches, evidencing the necessity of an analysis of the role of the agents of the triple helix in this scenario. The government is involved through the creation of policies to foster innovation, the provision of credits and incentives for the development of new products and the strengthening of MSE; Likewise, institutions of science and technology, including universities, play an extremely 
important role in strengthening this market, not only through basic research and the supply of intellectual capital to the market, but also by promoting practices for optimizing available resources and processes. The sharing of research infrastructures by Science and Technology Institutions may in conjunction with government policies reduce the cost with: a) acquisitions of equipment; b) physical and structural adequacy; c) training and management of intellectual capital; d) preventive maintenance.

As a result of the present study it was verified a lack of incentive programs focusing on the phases related to the scheduling of a technology (4 to 7 - TRL). As well as a high rate of interruption of scientific research due to the cost of acquisition and maintenance of R\&D equipment.

Existing government incentive programs have a priority focus on fostering basic research, leaving open the possibilities of studying new sharing models that result in a greater interaction between the triple helix agents and consequently greater efficiency in the conversion of basic research into new technologies for the market.

Although much has been done to foster research and development of technologies, the present study demonstrated that the current model of cooperation between triple helix agents has sought to keep up with the market changes that have occurred over the centuries. The university is no longer an external market agent with the sole purpose of generating theoretical knowledge. Leading universities have sought greater interaction with the market, developing knowledge and also technologies focused primarily on the development of basic research, anyway. Likewise, the government and research centers seek to adapt to this new economic reality with a focus on sustainable development and sharing economy. There is great market potential for the development of partnerships for the scheduling of technologies among triple-helix agents, which models require more studies.

\section{REFERENCES}

[1] House of commons science and technology committee. Bridging the valley of death: improving the commercialisation of research. United Kingdom, 2013.

[2] FILION, L. J. The Strategy of Successful Entrepreneurs in Small Business: Vision, Relationships and Antecipatory Learning. Ph.D. Thesis, University of Lancaster, 1988.

[3] SEBRAE - Serviço Brasileiro de Apoio às Micro e Pequenas Empresas. Análise do CAGED, 2017.

[4] PAVITT, K., ROBSON, M., TOWNSEND, J. The Size Distribution of Innovating Firms in the UK: 1945-1983. Journal of Industrial Economics. 35(3). 297-316, 1987.

[5] SCHUMPETER, J. The theory of economic development. Harvard University Press, Cambridge, 1934.

[6] DEMANTOVA NETO, Carlos; LONGO, Rose Maryjuliano. A gestão do conhecimento e a inovação tecnológica. Transinformação, Campinas, v. 13, n. 2, p. 93-110, Dec. 2001.

[7] BROWN, Rick. Managing Technological Innovation, Technology Strategies, Revista de Administração de Empresas 30, p.23-30, abr.1993.

[8] BARBIERl, José Carlos. Produção e Transferência de TecnoIogia. I. ed. São Paulo: Editora Ática, 1990. 